\title{
Delito de pornografía infantil una realidad escolar y comunitaria
}

\section{Child pornography crime a school and community reality}

\author{
Andrea Carolina Cruz Céspedes \\ andre caro16@hotmail.com \\ Universidad Técnica de Ambato, Ambato \\ Ecuador \\ https://orcid.org/0000-0001-8657-4176
}

Recibido: 1 de mayo de 2019

Aprobado: 13 de junio de 2019

\section{RESUMEN}

Se realizó un artículo de revisión con la finalidad de conocer la actualidad relacionada al delito de pornografía infantil una realidad escolar y comunitaria. Es importante destacar que el internet y redes sociales, son el cultivo perfecto para la generación de delitos relacionados a la pornografía infantil y abuso sexual, transcendiendo las fronteras del Ecuador, reseñándose como un delito de connotación global en donde los abusadores, toman diversas posiciones para poder cometer su fechoría sin ser detectados por las autoridades, familias e incluso por la propia víctima. Los llamados Grooming, Sexting, cyberbullying, son algunas modalidades relacionadas a la pornografía infantil desde el internet y redes sociales. Aunque en el Ecuador existen leyes y programas para la prevención del delito de pornografía infantil, surge la necesidad de implementar acciones de mayor contundencia para el resguardo de la infancia.

Descriptores: Abuso de menores; Pornografía; Problema social; Delincuencia; Internet.

\begin{abstract}
A review article was made in order to know the news related to the crime of child pornography a school and community reality. It is important to highlight that the internet and social networks are the perfect cultivation for the generation of crimes related to child pornography and sexual abuse, transcending the borders of Ecuador, being described as a crime of global connotation where abusers take various positions to to commit his
\end{abstract}




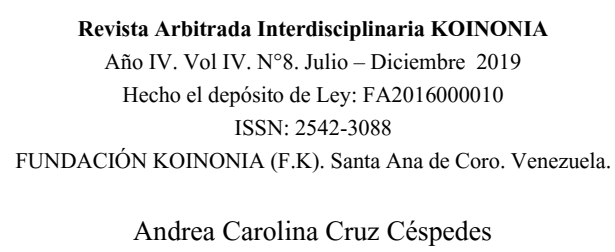

wrongdoing without being detected by the authorities, families and even by the victim himself. The so-called Grooming, Sexting, cyberbullying, are some modalities related to child pornography from the internet and social networks. Although in Ecuador there are laws and programs for the prevention of the crime of child pornography, there is a need to implement actions of greater force to protect children.

Descriptors: Child abuse; Pornography; Social problems; Delinquency; Internet.

\section{INTRODUCCIÓN}

La pornografía infantil en ámbitos escolares y comunitarios, representa uno de los flagelos globales a los cuales se ve amenazada la sociedad moderna, el uso de la internet y redes sociales, desde una óptica no guiada correctamente, puede incidir en la generación de interés por asumir conductas que atenten contra el pudor, sexualidad, de los infantes, las (Naciones Unidas , 1990) en la convención sobre los derechos del niño, en sus artículos 16 y 34, reglamenta el cuidado que debe existir sobre los infantes, respetándose su derecho al cuidado sexual, siendo los Estados firmantes de la declaratoria, responsables de generar los mecanismos necesarios para promover en asertividad, las condiciones que permitan velar por el cuidado integral de los niños.

Esto ha conllevado el fomento de la campaña "abre los ojos" (UNICEF Ecuador, 2018), lo cual permite sensibilizar a la sociedad sobre la importancia de proteger a la infancia de los abusos sexuales y al trabajo infantil, siendo indispensable además acotar el fomento de la campaña que impulsa la sexualidad responsable (Secretaria Nacional de Comunicación, 2018).

Por otro lado (Alba, 2017), "afirma que la educación sexual empieza en el hogar con los padres diciéndoles que nadie puede tocar sus partes íntimas: "Esto cuando tienen 2, 3 años y notamos que tienen más facilidades de comprender lo que está a su alrededor" (p. 1). Esto implica que se hacen esfuerzos por promover una educación sexual responsable, sin embargo, es necesario promover esta acción a fin de evitar que la pornografía infantil se constituye en una problemática social que dificulte el establecimiento de una interrelación saludable en la sociedad, por tal motivo la actual 


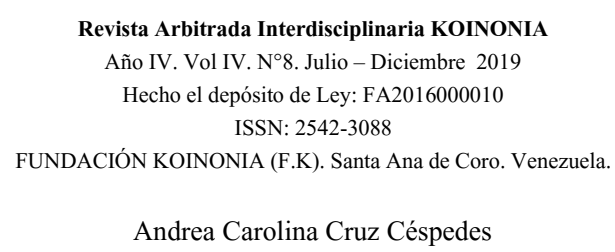

investigación hace una revisión de la tendencia sobre la pornografía infantil en contextos escolares y comunitarios, esto con la finalidad de alertar y advertir posibles escenarios sobre la educación en sexualidad de cara a los retos educativos del 2030 para el Ecuador.

\section{Pornografía escolar en la escuela y comunidad}

La pornografía infantil podría ser generada por diversos motivos, entre los cuales podría ser por prácticas de descubrimiento sexual que conlleven a la alternación del pudor cuando se trata con niños, existiendo así eventos como la pedofilia, definida por (American Psychiatric Association, 1995).

La característica esencial de la pedofilia supone actividades sexuales con niños pre púberes (generalmente de 13 o menos años de edad). El individuo con este trastorno debe tener 16 años o más y ha de ser por lo menos 5 años mayor que el niño. En los adolescentes mayores que presentan este trastorno no se especifica una diferencia de edad precisa, y en este caso debe utilizarse el juicio clínico; para ello, debe tenerse en cuenta tanto la madurez sexual del niño como la diferencia de edades. La gente que presenta pedofilia declara sentirse atraída por los niños dentro de un margen de edad particular. Algunos individuos prefieren niños; otros, niñas, y otros, los dos sexos (p. 540).

Este tipo de actividad sexual, podría ocurrir cuando la persona posee trastornos psiquiatrías o psicológicos que potencian la atracción sexual con niños o niñas, siendo esta situación tipificada como delito en la (Constitución del Ecuador, 2008), articulo 66, numeral 3, y en el Artículo 178 del (Código Orgánico Integral Penal, 2014); así como atentar contra las normas socialmente establecidas por la sociedad ecuatoriana. Se hace necesario que se generen mecanismos en el sistema educativo donde se aborde periódicamente la salud mental de las personas que forman parte de la institución educativa, esto podría prevenir la generación de abusos infantiles en recintos escolares. Aunado a lo planteado, (Ecuavisa, 2019), reseña la detención de un profesor en lbarra Ecuador, acusado de pertenecer a una red de pornografía internacional, decomisándole videos e niños, niñas y adolescentes, estudiantes de la institución educativa donde labora; lo cual atenta contra la integridad de los menores involucrados, produciendo 
alternaciones negativas en su personalidad que pueden tener connotación en su conducta, siendo necesaria la adecuada atención psicológica por especialistas, así como el acompañamiento familiar.

Este delito ha sido observado a nivel de bandas, lo que invade la privacidad de los infantes, no solo en la escuela, sino en su contexto comunitario, (Ecuavisa, 2019), lo cual amerita coordinar entre los organismos de seguridad y sector escolar, campañas de prevención sobre el abuso infantil en todas sus modalidades, en especial al sexual, siendo necesario identificar factores amenazantes como:

\section{Internet y sexo}

El internet como herramienta de comunicación global ha revolucionado la vida global de las personas, siendo necesaria generar una adecuada educación en el hogar a los niños sobre el uso adecuado de la tecnología, por cuanto la educación ha adoptado como apoyo vital el uso de las TIC (tecnologías de la información y comunicación), como recursos pedagógicos con la finalidad de estar en concordancia con las tendencias en la cuales se enmarca la sociedad, sin embargo, no todo el uso a la internet y TIC, resulta con fines educativos, (Ecuavisa, 2019), genera un reportaje donde se advierte el peligro que representan las plataformas de video juegos, por cuanto pedófilos podrían estar escondidos en seudónimos de jugadores con la finalidad de atraer niños con el propósito de violentar su sexualidad.

El tema de la pornografía y del internet, permite identificar modus operandi de personas inescrupulosas que se esconden detrás de fachadas inocentes, siendo necesario que padres, representantes, docentes, estén al tanto de los video juegos y acceso al internet de sus hijos o estudiantes, esto podría conformar la posibilidad de promover acciones favorables en evitar delitos de pornografía infantil. El delito pornográfico por internet no es novedoso, de acuerdo a (Morales, 2001):

La pornografía infantil constituye un problema de dimensión internacional, que se ha amplificado con la irrupción de nuevas tecnologías que han transformado las pautas de producción y difusión de este tipo de material. La 


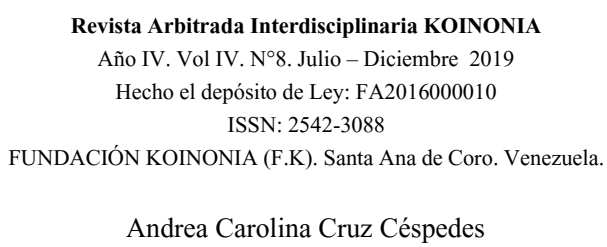

Andrea Carolina Cruz Céspedes

transformación de la producción y difusión de la pornografía infantil en general, y particularmente aprovechando el nuevo escenario que facilitan las nuevas tecnologías, abre interrogantes al Derecho Penal de diversa consideración (p. 1).

Se recomienda generar una educación preventiva y formativa de una identidad sexual que favorezca el crecimiento integral de los estudiantes en conformidad a una conducta bioética basada en un proyecto de vida, lo cual permite direccionalidad en el niño en la construcción de una personalidad cónsona a los principios de convivencialidad aceptados por la sociedad ecuatoriana. (Hernández, 2013), sostiene que el internet constituye una amplia red de posibilidades de generar conflictos de índole sexual a los menores de edad, por cuanto permite generar interconectividad con las redes sociales, generándose modalidades de acecho sexual, entre las cuales se encuentran:

1. Grooming: conjunto de estrategias que una persona adulta desarrolla para ganarse la confianza del menor a través de Internet con el fin último de obtener concesiones de índole sexual. Hablamos entonces de acoso sexual a menores en la Red y el término completo sería child grooming o internet grooming. Desde un acercamiento lleno de empatía y/o engaños se pasa al chantaje más cruento para obtener imágenes comprometidas del menor y, en casos extremos, pretender un encuentro en persona. El daño psicológico que sufren niños, niñas y adolescentes atrapados en estas circunstancias es enorme (Flores Fernández, 2008).

2. Sexting: consiste en enviar textos 0 imágenes insinuantes, eróticos 0 pornográficos por Internet o por los teléfonos móviles. Es una práctica bastante extendida entre los adolescentes que por iniciativa propia suelen enviar mensajes sexting a sus novios/as como prueba de afecto, a alguien con quieren ligar, como broma, etc. El problema es que ese texto o imagen puede ser utilizada más tarde por el destinatario u otro desconocido para extorsionar o chantajear a la víctima. Esto se conoce con el nombre de extorsión (Gobierno de Canarias, 2014).

Las prácticas indicadas, progresivamente toman auge en la sociedad global, proyectándose a encriptarse como un flagelo que puede traer además consecuencias 
como el bullying sexual (Mejía-Soto, 2014), señala que "A largo plazo, las consecuencias de sufrir este tipo de bullying pueden establecer el desarrollo de casos de estrés postraumático". El tema alude a la necesidad de generar programas de formación en las escuelas y comunidades con la finalidad de advertir sobre estas tipologías de violencia sexual que podrían estar incentivando a la pornografía infantil, otros de los problemas a atender es el referido al cyberbullying (Revista Cabal, 2010), indica diversos indicadores que deben tener en cuenta los padres con la finalidad de prevenir el cyberbullying:

1. Evitar que, en lo posible, el chico tenga una cuenta propia en una red social. Aunque la mayor parte de los menores que viven en las grandes ciudades cuentan con una cuenta propia, la edad de 13 años es uno de los requisitos necesario para abrir una cuenta de correo electrónico o en una red social.

2. Sume a su hijo como su amigo en la red social. Eso le permitirá ejercer cierto grado de control sobre lo que hace, comenta o pública. Cuando él tenga su propio correo electrónico, el pacto puede ser que usted también disponga de la contraseña de acceso. Explíquele la necesidad de ser cuidadoso en el manejo de estas herramientas.

3. Establezca las bases para una buena comunicación con su hijo. Recuerde que usted está a cargo de su cuidado y educación, y explíquele que si alguien lo acosa -en persona o en Internet él debe decírselo cuanto antes, para que usted pueda tomar las medidas necesarias para protegerlo. Es conveniente aclarar de ante mano que sufrir alguna agresión o acoso no es algo que a él deba avergonzarlo.

4. Limite el tiempo que su hijo emplea en Internet o chateando con sus amigos. Se pueden pautar determinadas horas por día o por semana. Lo importante es que no tenga acceso irrestricto a las redes, en cualquier momento y horario.

5. Evite que su hijo tenga computadora en su dormitorio. Cuanto más tiempo pasen los hijos en presencia de sus padres, menos probabilidades tendrán de meterse en problemas en Internet. Lo ideal es tener una computadora familiar, en algún 
lugar de la casa como el living o el comedor que todos utilicen, de manera que la privacidad quede acotada a otras prácticas y no al uso de las redes sociales.

6. Predique con el ejemplo. Si sus hijos lo ven enviando mensajes de texto o hablando por celular a toda hora, e incluso usando Facebook con excesiva frecuencia, esto les parecerá lo más normal del mundo. Internet es una gran fuente de información y socialización, pero es importante aprender a equilibrar su uso.

7. Observe el comportamiento y la actitud de tu hijo. Aunque crea que su hijo le cuenta todo, no de esto por hecho. Si lo nota más triste de lo habitual, o percibe un menor rendimiento escolar, indague en las causas del cambio de comportamiento. Explíquele que no debe tener miedo de contarle si alguien lo está molestando o agrediendo.

8. Si su hijo es víctima de acoso, tome acción de inmediato. Hable con los maestros, cambie el número de teléfono de su hijo, cierre su cuenta de correo electrónico y su cuenta de red social, e incluso informe a las autoridades si es preciso. El bullying ha costado la vida a pre-adolescentes y adolescentes y hay que tomarlo en serio.

9. Involúcrese en su vida social. Conozca a sus maestros, a sus compañeros de escuela, a sus amigos y a los padres de sus amigos. Está muy bien lo de permitir que tenga privacidad, pero cuando sea adulto. La niñez, la pre-adolescencia y la adolescencia son momentos de saber siempre con quién anda y qué está haciendo.

10. Ayúdele a tener confianza en sí mismo. Los niños tímidos, acomplejados o con alguna diferencia física, étnica, o social, tienen más tendencia a ser víctimas del acoso escolar o cibernético. En caso de que su hijo pueda sentirse diferente a sus amigos, ayúdelo a desarrollar confianza en sí mismo mediante el deporte, la pertenencia a un club o la práctica de algún hobby que él disfrute.

Los padres deben desarrollar empatía con sus hijos con la finalidad de poder tener comunicación asertiva, de ese modo, no percibirán que están siendo controlados 
excesivamente, generando un clima de confianza que permita hablar de temas de educación en sexualidad, incluyendo la pornografía infantil, por cuanto no solo puede darse el hecho de que sean inducidos por terceras personas, sino, que el hecho de crecer y querer explorar, puede conducir a ver pornografía (Orgaz-Baz, 2013), señala que esta situación es propia de la etapa de descubrimiento sexual, (González, 2019), complementa que la sociedad transita entre la protección del niño y la hipersexualidad originada por la sociedad neoliberal que contribuye a hiperexcitación en los infantes.

Otro factor a tener en consideración es lo referido al debut sexual del infante (Martínez, 2018), esto inicia con la auto exploración del infante, entre los dos y tres años, etapa donde puede tocarse sus parte intimas sin tener deseo o satisfacción, siendo pertinente que los padres tengan claridad sobre el tema, sobre todo porque permite educarse en sexualidad con la finalidad de prevenir encuentros no permitidos entre un adulto y el infante, por cuanto suele ocurrir que el infante pueda estar en contacto con personas enfermas psicológicamente en relación al manejo asertivo de la sexualidad, pudiéndose aprovechar de la auto exploración del niño con el fin de realizar actos lascivos. (García \& García, 2018), señalan:

En torno a los 3 ó 4 años puede mostrar interés por los genitales de otros niños o de sus padres. Pueden tocar los pechos de mujeres o mirar a adultos desnudos. Empiezan a hacer preguntas sobre el porqué de las diferencias entre sexos y sobre la reproducción y el origen de los bebés. Observan con atención lo que sucede entre los adultos que los rodean. Es normal que imiten comportamientos y que inicien los juegos sexuales infantiles como besarse o cogerse de las manos, jugar "a médicos" o "a papás y mamás".

Como se ha indicado, es necesario educar a los adultos sobre el manejo de la sexualidad por parte de infante, esto le permitirá conocer cuales son las conductas naturales para evitar caer en escándalos o tabús que impiden un sano desarrollo, así como también identificar quienes están a su alrededor con la finalidad de evitar desconocidos e incluso familiares que pudiesen desarrollar abusos contra el infante por el simple hecho de confundir la exploración del infante con la búsqueda de respuestas sexuales, siendo esto 
posible por cuanto en el ser humano se generan vinculaciones afectivas con sus semejantes e incluso mascostas.

\section{Manifestaciones saludables de la sexualidad infantil.}

\section{Figura 1.}

Relacionadas con el género

Curiosidad y preguntas frecuentes sobre la diferencia entre niños y niñas

\begin{tabular}{|c|c|}
\hline & $\begin{array}{l}\text { llaman la atención sus diferencias } \\
\text { - Juegan al papá y a la mamá } \\
\text { - Niñas y niños se visten con ropas de los progenitores, independientemente del sexo } \\
\text { - Juegan y pueden vestirse o expresarse como personas del sexo opuesto } \\
\text { - Curiosidad por observar o tocar el cuerpo de los adultos }\end{array}$ \\
\hline $\begin{array}{l}\text { Vínculos } \\
\text { afectivos }\end{array}$ & $\begin{array}{l}\text { - Interés por tener novio o novia (con amigos, familiares o personajes favoritos) } \\
\text { - Curiosidad por el matrimonio (las personas cercanas o queridas son elegidas como "novio" } \\
\text { o "esposo") } \\
\text { - Interés por crecer, enamorarse y tener una pareja } \\
\text { - Sufren por rechazos afectivos entre sus iguales } \\
\text { - Cuidan, alimentan y brindan afecto a sus juguetes y mascotas } \\
\text { - Interés o preocupación por el divorcio o separación de los progenitores } \\
\text { - Temor sobre la pérdida de seres queridos }\end{array}$ \\
\hline Erotismo & $\begin{array}{l}\text { - Estimular sus genitales con la mano o frotándolos con objetos, muebles o juguetes } \\
\text { Juegos sexuales: niños y niñas de edad similar juegan a acercar sus cuerpos para verlos y } \\
\text { tocarlos } \\
\text { - Se abrazan y besan entre edades similares } \\
\text { - Juegan al doctor, los esposos, exploran su cuerpo y los genitales }\end{array}$ \\
\hline Reproducción & $\begin{array}{l}\text { Juegan o imitan ser como papá o como mamá } \\
\text { - Juegan a la "casita" y a tener una familia } \\
\text { - } \quad \text { Expresan su deseo de convertirse en padre o madre } \\
\text { - Realizan funciones de padre o madre: cuidar o proteger a mascotas, muñecos u otros niños } \\
\text { - } \quad \text { Expresan su deseo de tener un bebé } \\
\text { - Preguntas frecuentes sobre el matrimonio, el origen y nacimiento de los bebés }\end{array}$ \\
\hline
\end{tabular}

Tomado de (García-Piña, 2016).

Sin embargo, cuando el niño es abusado sexualmente, aunado a la presencia de pornografía, varía su comportamiento consigo mismo y con los demás, (Familydoctor.org, 2017), indica que:

Cualquier actividad sexual con un niño (por un adulto u otro niño) constituye abuso sexual. Causa dolor físico y emocional. Tiene efectos a largo plazo en el niño. $Y$ es un delito. Reconocer el abuso sexual infantil no es fácil. Los niños tienen miedo y sienten vergüenza de contarlo. Muchas veces, el abusador es alguien que el niño conoce y en quien confía. Podría ser un miembro de la familia o alguien que vive en la casa del niño. También puede ser un extraño (p. 1). 
Por lo tanto, el infante al ser abusado experimenta cambios negativos en su conducta y personalidad, siendo pertinente que los padres al tener conocimiento del abuso, le brinden afecto y confianza, lo cual es un paso importante para acceder a ayuda especializada en contribución de superar el trauma ocasionado en el menor. (Navas, 2014), señala que el:

El abuso sexual en menores de edad es uno de los tipos de maltrato infantil con peores repercusiones en sus víctimas y que usualmente coexiste con otros tipos de violencia. Incluye tanto agravios que no involucran contacto físico como aquellos que sí lo hacen (p. 2).

El abuso sexual y la pornografía infantil son generadores de una serie de alternaciones en el infante, siendo esto perjudicial para su sano desarrollo integral, siendo necesaria la debida prevención por parte del Estado - familia y comunidad, como factores protectores, de ese modo (Aular, 2016), explica la importancia de la familia y de la educación en la contribución de una infancia saludable, (Aldana Zavala \& Valles González, 2018), indican que la mujer juega un papel fundamental en la formación de niños saludables, siendo indispensable que la propia mujer no haya sido abusada o violentada, con la finalidad de formar hogares asertivos.

\section{REFLEXIONES DE CIERRE}

La pornografía infantil es un delito en pleno auge en el Ecuador, se han citado casos desarrollados en la escuela y en entornos comunitarios, de ese modo, urge la necesidad de estructurar planes preventivos y formativos desde la escuela como escenario integrador de la sociedad, para lo cual se requiere que el Estado, familia y el sistema educativo en todos sus niveles, interactúen entre sí, para acceder a políticas que permitan generar acciones favorables en beneficio de la infancia.

Es importante destaca que el internet y redes sociales, son el cultivo perfecto para la generación de delitos relacionados a la pornografía infantil y abuso sexual, transcendiendo las fronteras del Ecuador, reseñándose como un delito de connotación 


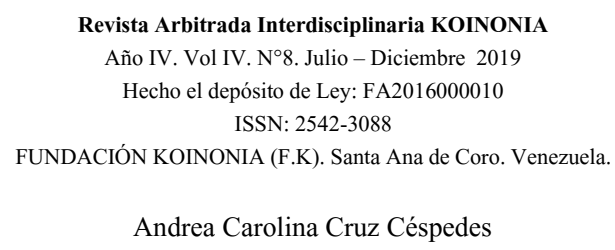

global en donde los abusadores, toman diversas posiciones para poder cometer su fechoría sin ser detectados por las autoridades, familias e incluso por la propia víctima. Los llamados Grooming, Sexting, cyberbullying, son algunas modalidades relacionadas a la pornografía infantil desde el internet y redes sociales, destacándose que quienes utilizan este tipo de elementos, son personas enfermas en lo psiquiátrico - psicológico, por lo que no puede concebirse ingenuamente la pornografía infantil como hechos aislados, al contrario, existen redes organizadas para el desarrollo del delito de pornografía infantil.

Aunque en el Ecuador existen leyes y programas para la prevención del delito de pornografía infantil, surge la necesidad de implementar acciones de mayor contundencia para el resguardo de la infancia, debido que esta por ser una población vulnerable, es captada con mayor facilidad por los delincuentes, aunado que un infante abusado, proyecta una personalidad trastocada que debe ser atendida pertinente por los profesionales de la salud, por cuanto no hacerlo, posibilita una cadena delincuencial relacionada a la pornografía y abuso sexual.

\section{REFERENCIAS CONSULTADAS}

1. Alba, B. (2017). La educación sexual tiene debilidades en Ecuador. Obtenido de https://www.eluniverso.com/noticias/2017/10/29/nota/6455173/educacionsexual-tiene-debilidades-ecuador

2. Aldana Zavala, J., \& Valles González, E. (2018). La mujer en Derecho a una Vida libre de violencia desde la Organización Comunitaria. IUSTITIA SOCIALIS, 3(5), 110-127. doi:http://dx.doi.org/10.35381/racji.v3i5.171

3. Association, A. P. (1995). Pedofilia. En Manual diagnóstico y estadísticode los trastornos mentales IV (pág. 540). Barcelona, España: Impresión: Gràfiques 92, S.A. - Torrassa, $108 \mathrm{E}$ - Sant Adrià de Besòs (1995). recuperado de https://psicovalero.files.wordpress.com/2014/06/manual-diagnc3b3stico-yestadc3adstico-de-los-trastornos-mentales-dsm-iv.pdf

4. Aular, M. R. (2016). Programa de estrategias pedagógicas dirigido a los docentes para la participación de la familia al proceso de aprendizaje en la 


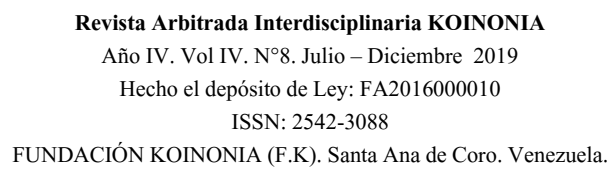

Andrea Carolina Cruz Céspedes

atención educativa no convencional. Revista Arbitrada Interdisciplinaria Koinonía, 50-73. Obtenido de http://fundacionkoinonia.com.ve/ojs/index.php/revistakoinonia/article/view/39/2 $\underline{7}$

5. Código Orgánico Integral Penal. (2014). Registro Oficial . Obtenido de https://tbinternet.ohchr.org/Treaties/CEDAW/Shared\%20Documents/ECU/INT CEDAW ARL ECU 18950 S.pdf

6. Constitución del Ecuador. (2008). Constitución del Ecuador. Obtenido de Constitución del Ecuador: https://www.oas.org/juridico/mla/sp/ecu/sp ecu-inttext-const.pdf

7. Ecuavisa. (27 de junio de 2019). Desarticulan 11 bandas delictivas en 8 provincias. Se dedicaban al tráfico de drogas y pornografía infantil. Quito, Pichincha, Ecuador. Obtenido de https://www.ecuavisa.com/articulo/noticias/nacional/503671-desarticulan-11bandas-delictivas-8-provincias

8. Ecuavisa. (6 de 09 de 2019). Detienen en Ibarra a profesor acusado de pornografía infantil. Ibarra, Imbabura, Ecuador. Obtenido de https://www.ecuavisa.com/articulo/noticias/nacional/526601-detienen-ibarraprofesor-acusado-pornografia-infantil

9. Ecuavisa. (31 de mayo de 2019). Pensaba que mi hijo jugaba a un videojuego inocente. Roblox es una plataforma donde los jugadores pueden elegir su avatar. Quito, Pichincha , Ecuador. Obtenido de https://www.ecuavisa.com/articulo/noticias/internacionales/494656-pensabaque-mi-hijo-jugaba-videojuego-inocente

10. Familydoctor.org. (2017). Los signos de abuso sexual en la infancia. Obtenido de https://es.familydoctor.org/los-signos-de-abuso-sexual-en-la-infancia/

11. Fernández, J. F. (2008). Grooming, acoso a menores en la Red. Obtenido de https://www.pantallasamigas.net/grooming-acoso-a-menores-en-la-red/

12. García-Piña, C. A. (2016). Sexualidad infantil: información para orientar la práctica clínica. Acta Pediatr $\operatorname{Mex}(37)$, 47-53. Obtenido de http://www.scielo.org.mx/pdf/apm/v37n1/2395-8235-apm-37-01-00047.pdf 


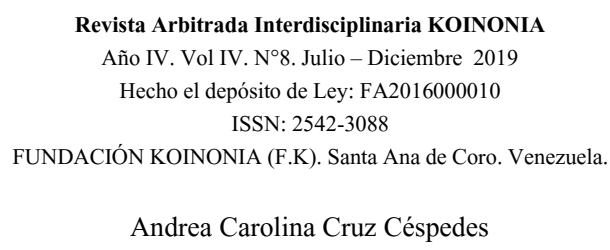

Andrea Carolina Cruz Céspede

13. Gobierno de Canarias. (2014). 2.3 Sexting. Obtenido de http://www3.gobiernodecanarias.org/medusa/contenidosdigitales/FormacionTI C/cdtic2014/03co/23 sexting.html

14. González, P. A. (2019). Erotización infantil y gramáticas afectivas: discursos sobre la infancia en la era 2.0 en Argentina. Sexualidad, Salud y Sociedad, 101 118. Obtenido http://www.scielo.br/scielo.php?script=sci arttext\&pid=S1984$\underline{64872019000100101 \& \operatorname{lng}=\mathrm{pt} \& \mathrm{nrm}=\mathrm{iso}}$

15. Hernández, G. G. (2013). La protección de la infancia frente a la pornografía infantil. luris Tantum Revista Boliviana de Derecho(15), 90-110. Obtenido de http://www.scielo.org.bo/scielo.php?script=sci arttext\&pid=S207081572013000100006

16. Martínez, R. L. (2018). Factores asociados al debut sexual, actividad sexual en línea y calificación en estudiantes de Morelia. RESPYN Revista Salud Pública y Nutrición, 16-22. doi:https://doi.org/10.29105/respyn17.1-3

17. Mejía-Soto, G. (2014). Sexting: una modalidad cada vez más extendida de violencia sexual entre jóvenes. Perinatología y reproducción humana, 217-221. Obtenido http://www.scielo.org.mx/scielo.php?script=sci arttext\&pid=S0187$\underline{53372014000400007}$

18. Morales, F. (2001). Pornografía infantil e internet. Obtenido de https://www.uoc.edu/in3/dt/20056/index.html

19. Naciones Unidas. (1990). Convención sobre lo derechos del niño . Obtenido de UNICEF Ecuador: https://www.unicef.org/ecuador/convencion 2.pdf

20. Navas, M. J. (2014). Abuso sexual en menores de edad: generalidades, consecuencias y prevención. Medicina Legal de Costa Rica, 31(1), 2-13. Obtenido de https://www.scielo.sa.cr/pdf/mlcr/v31n1/art06v31n1.pdf

21. Orgaz-Baz, E. G.-Q. (2013). La exposición de los menores a la pornografía en Internet: prevalencia, motivaciones, contenidos y efectos. Anales de Psicología, 319-327.

Obtenido de http://scielo.isciii.es/scielo.php?script=sci abstract\&pid=S0212$\underline{97282013000200003 \& \operatorname{lng}=\text { es\&nrm=iso }}$ 


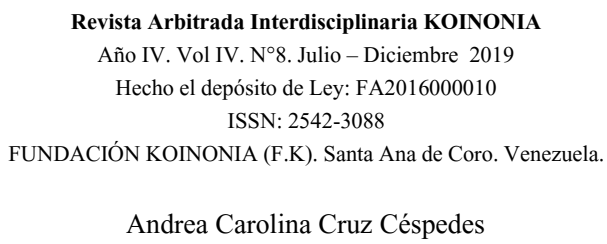

22. Revista Cabal. (2010). Cyberbullying: el acoso a través de las redes sociales. Obtenido de http://www.revistacabal.coop/actualidad/cyberbullying-el-acosotraves-de-las-redes-sociales

23. Saseta, P. G. (2018). Desarrollo de la sexualidad en el niño preescolar. Obtenido de http://www.familiaysalud.es/podemos-prevenir/eventos-deldesarrollo/consejo-anticipado/desarrollo-de-la-sexualidad-en-el-nino

24. Secretaria Nacional de Comunicación. (2018). En Ecuador se impulsa la educación sexual responsable. Obtenido de Secretaría general de la comunicación de la presidencia: https://www.comunicacion.gob.ec/en-ecuadorse-impulsa-la-educacion-sexual-responsable/

25. UNICEF Ecuador. (2018). Campaña "Abre los Ojos" contra el abuso sexual lanza UNICEF en Ecuador. Obtenido de https://www.unicef.org/ecuador/spanish/media 5606.htm

\section{REFERENCES CONSULTED}

1. Alba, B. (2017). Sex education has weaknesses in Ecuador. Retrieved from https://www.eluniverso.com/noticias/2017/10/29/nota/6455173/educacion$\underline{\text { sexual-tiene-debilidad-ecuador }}$

2. Aldana Zavala, J., \& Valles González, E. (2018). Women in Right to a Life free of violence from the Community Organization. IUSTITIA SOCIALIS, 3 (5), 110127. doi: http: //dx.doi.org/10.35381/racji.v3i5.171

3. Association, A. P. (1995). Pedophilia In Diagnostic and Statistical Manual of Mental Disorders IV (p. 540). Barcelona, Spain: Printing: Gràfiques 92, S.A. Torrassa, 108 E - Sant Adrià de Besòs (1995). Retrieved from https://psicovalero.files.wordpress.com/2014/06/manual-diagnc3b3stico-yestadc3adstico-de-los-trastorno-mentales-dsm-iv.pdf

4. Aular, M. R. (2016). Program of pedagogical strategies aimed at teachers for the participation of the family to the learning process in unconventional educational care. Interdisciplinary Arbitrated Magazine Koinonía, 50-73. Retrieved

from http://fundacionkoinonia.com.ve/ojs/index.php/revistakoinonia/article/view/39/2 $\underline{7}$ 


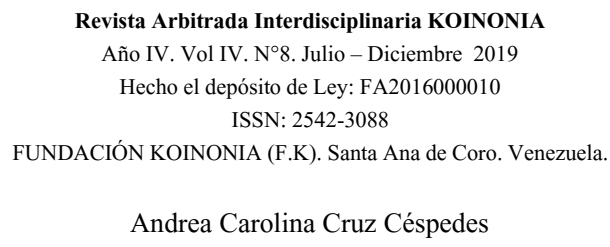

5. Organic Integral Criminal Code. (2014). Official register . Retrieved from https://tbinternet.ohchr.org/Treaties/CEDAW/Shared\%20Documents/ECU/INT CEDAW ARL ECU 18950 S.pdf

6. Constitution of Ecuador. (2008). Constitution of Ecuador. Obtained from the Constitution of Ecuador: https://www.oas.org/juridico/mla/sp/ecu/sp ecu-inttext-const.pdf

7. Ecuavisa. (June 27, 2019). They dismantle 11 criminal gangs in 8 provinces. They engaged in drug trafficking and child pornography. Quito, Pichincha, Ecuador. Retrieved from https://www.ecuavisa.com/articulo/noticias/nacional/503671-desarticulan-11bandas-delictivas-8-provincias

8. Ecuavisa. (6 of 09 of 2019). Ibarra arrested a professor accused of child pornography. Ibarra, Imbabura, Ecuador. Retrieved from https://www.ecuavisa.com/articulo/noticias/nacional/526601-detienen-ibarraprofesor-acusado-pornografia-infantil

9. Ecuavisa. (May 31, 2019). I thought my son played an innocent video game. Roblox is a platform where players can choose their avatar. Quito, Pichincha, Ecuador. Retrieved from https://www.ecuavisa.com/articulo/noticias/internacionales/494656-pensabaque-mi-hijo-jugaba-videojuego-inocente

10. Familydoctor.org. (2017). Signs of sexual abuse in childhood. Retrieved from https://es.familydoctor.org/los-signos-de-abuso-sexual-en-la-infancia/

11. Fernández, J. F. (2008). Grooming, harassment of minors on the Web. Obtained from https://www.pantallasamigas.net/grooming-acoso-a-menoresen-la-red/

12. García-Piña, C. A. (2016). Child sexuality: information to guide clinical practice. Acta Pediatr Mex (37), 47-53. Retrieved from http://www.scielo.org.mx/pdf/apm/v37n1/2395-8235-apm-37-01-00047.pdf

13. Government of the Canary Islands. (2014). 2.3 Sexting Retrieved from http://www3.gobiernodecanarias.org/medusa/contenidosdigitales/FormacionTI C/cdtic2014/03co/23 sexting.html

14. González, P. A. (2019). Child eroticization and affective grammars: speeches about childhood in the 2.0 era in Argentina. Sexuality, Health and Society, 101- 
118.

Retrieved

http://www.scielo.br/scielo.php?script=sci arttext\&pid=S1984-

$\underline{64872019000100101 \& \operatorname{lng}=\mathrm{pt} \& \mathrm{nrm}=\mathrm{iso}}$

15. Hernández, G. G. (2013). Childhood protection against child pornography. luris Tantum Bolivian Journal of Law (15), 90-110. Retrieved from http://www.scielo.org.bo/scielo.php?script=sci arttext\&pid=S2070$\underline{81572013000100006}$

16. Martínez, R. L. (2018). Factors associated with sexual debut, online sexual activity and qualification in Morelia students. RESPYN Public Health and Nutrition Magazine, 16-22. doi: https: //doi.org/10.29105/respyn17.1-3

17. Mejía-Soto, G. (2014). Sexting: an increasingly widespread modality of sexual violence among young people. Perinatology and human reproduction, 217-221. Retrieved http://www.scielo.org.mx/scielo.php?script=sci arttext\&pid=S0187$\underline{53372014000400007}$

18. Morales, F. (2001). Child pornography and internet. Retrieved from https://www.uoc.edu/in3/dt/20056/index.html

19. United Nations. (1990). Convention on the rights of the child. Obtained from UNICEF Ecuador: https://www.unicef.org/ecuador/convencion_2.pdf

20. Navas, M. J. (2014). Sexual abuse in minors: generalities, consequences and prevention. Legal Medicine of Costa Rica, 31 (1), 2-13. Retrieved from https://www.scielo.sa.cr/pdf/mlcr/v31n1/art06v31n1.pdf

21. Orgaz-Baz, E. G.-Q. (2013). The exposure of minors to pornography on the Internet: prevalence, motivations, content and effects. Annals of Psychology, 319-327.

Retrieved from http://scielo.isciii.es/scielo.php?script=sci abstract\&pid=S021297282013000200003\&lng=es\&nrm=iso

22. Cabal Magazine. (2010). Cyberbullying: harassment through social networks. Obtained from http://www.revistacabal.coop/actualidad/cyberbullying-el-acosotraves-de-las-redes-sociales

23. Saseta, P. G. (2018). Development of sexuality in the preschool child. Retrieved from http://www.familiaysalud.es/podemos-prevenir/eventos-deldesarrollo/consejo-anticipado/desarrollo-de-la-sexualidad-en-el-nino 
24. National Secretary of Communication. (2018). In Ecuador, responsible sex education is promoted. Obtained from the General Secretariat of the communication of the presidency: https://www.comunicacion.gob.ec/enecuador-se-impulsa-la-educacion-sexual-responsable/

25. UNICEF Ecuador. (2018). "Open your eyes" campaign against sexual abuse launches UNICEF in Ecuador. Retrieved from https://www.unicef.org/ecuador/spanish/media 5606.htm

(C2019 por el autore. Este artículo es de acceso abierto y distribuido según los términos y condiciones de la licencia Creative Commons Atribución-NoComercial-Compartirlgual 4.0 Internacional (CC BY-NC-SA 4.0)

(https://creativecommons.org/licenses/by-nc-sa/4.0/). 